\title{
CIP2A regulates proliferation and apoptosis of multiple myeloma cells
}

\author{
ZHUANZHEN ZHENG $^{1}$, ZHENHUA QIAO ${ }^{1}$, WENLIANG CHEN ${ }^{1}$, RONG GONG $^{2}$, YALIN WANG $^{3}$,

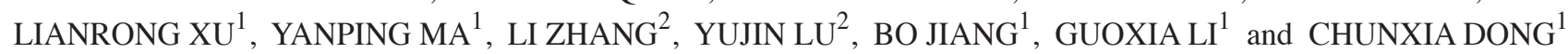 \\ ${ }^{1}$ Department of Hematology, The Second Hospital of Shanxi Medical University, Taiyuan, Shanxi 030001; \\ ${ }^{2}$ Shanxi Academy of Medical Sciences, Shanxi University Hospital, Taiyuan, Shanxi 030006; \\ ${ }^{3}$ Department of Hematology, The Second People's Hospital of Shanxi, Taiyuan, Shanxi 037008, P.R. China
}

Received May 7, 2015; Accepted April 2, 2016

DOI: $10.3892 / \mathrm{mmr} .2016 .5553$

\begin{abstract}
Multiple myeloma (MM) is one of the most common causes of mortality from hematological malignancy in China. Recent studies have demonstrated that cancerous inhibitor of protein phosphatase $2 \mathrm{~A}$ (CIP2A) may exhibit a role in promoting the growth of cancer; however, the function of CIP2A in $\mathrm{MM}$ remains unknown. In the present study, the expression and molecular mechanism underlying the effects of CIP2A in patients with $\mathrm{MM}$ and in MM cell lines were elucidated. Firstly, the expression of CIP2A was detected in patients with $\mathrm{MM}$ and in $\mathrm{MM}$ cell lines by reverse transcription-quantitative polymerase chain reaction. Furthermore, silencing of CIP2A with short hairpin RNA was performed in MM cells, and the impact on the proliferation and apoptosis of RPMI-8226 cells was analyzed (as endogenous CIP2A is highly expressed in RPMI-8226 cell lines compared with other cells). CIP2A was significantly elevated in patients with $\mathrm{MM}$ and in $\mathrm{MM}$ cell lines, and silencing of CIP2A could inhibit the proliferation ability of RPMI-8226 cells in vitro. In addition, CIP2A knockdown induced apoptosis and led to substantial reduction of c-Myc protein levels in MM cell lines. This study suggested that CIP2A inhibition may provide a promising therapeutic strategy for patients with MM.
\end{abstract}

\section{Introduction}

Multiple myeloma (MM) is a hematological malignancy derived from clonal B cells. Different stages of differentiation of the neoplastic clone may cause heterogeneity in MM (1). MM is relatively uncommon but can be fatal. Patients with MM initially respond to treatment, but eventually relapse and

Correspondence to: Professor Wenliang Chen, Department of Hematology, The Second Hospital of Shanxi Medical University, 382 Wuyi Road, Taiyuan, Shanxi 030001, P.R. China

E-mail: drchenwl6@163.com

Key words: multiple myeloma, proliferation, apoptosis, cancerous inhibitor of protein phosphatase $2 \mathrm{a}, \mathrm{c}-\mathrm{Myc}$, targeted therapy succumb to disease after several months or years $(2,3)$. Thus, the development of novel treatment strategies to effectively control MM are required.

Cancerous inhibitor of protein phosphatase 2A (CIP2A) is a novel human oncoprotein that can promote tumor transformation and maintain the malignant phenotype in various types of cancer (4). The oncogenic effects of CIP2A may occur in part through stabilization of c-Myc (5). CIP2A levels are significantly elevated in several malignancies, compared with normal tissue (6-8). To the best of our knowledge, CIP2A expression has not been reported in MM. Thus, the present study aimed to determine the levels of CIP2A in patients with $\mathrm{MM}$ and in MM cell lines. In addition to the effect of CIP2A on MM cell growth and apoptosis following knockdown using shRNA, phosphorylation of key signaling molecules was analyzed in vitro.

\section{Materials and methods}

Serum samples. In total, 40 serum samples were obtained from patients with MM who underwent treatment at the Second Hospital of Shanxi Medical University (Taiyuan, Shanxi) between October 2010 and August 2014 (20 cases with $\kappa$-type, 16 cases with $\lambda$-type and 4 cases with non-secretory type). There were 20 serum samples from the normal control without any detectable bone marrow abnormalities observed during physical examination. The controls had a median age of 42 years (range, 31-60 years), controls were excluded if they were deceased or had been previously diagnosed with a hematological malignancy at the corresponding MM patient diagnosis. The diagnostic criteria of all MM patients was determined using the International Myeloma Working Group 2012 system (9). This study was approved by the Ethics Committee of the Second Hospital of Shanxi Medical University. All patients provided informed consent.

Cell lines and cell culture. U266, KM3 and RPMI-8226 human myeloma cell lines were obtained from American Type Culture Collection (Manassas, VA, USA). All cells were maintained in RPMI-1640 medium (Gibco; Thermo Fisher Scientific, Inc., St. Louis, MO, USA) supplemented with $10 \%$ fetal bovine serum (FBS; Gibco; Thermo Fisher Scientific, Inc.) at $37^{\circ} \mathrm{C}$, in 
a humidified atmosphere containing $5 \% \mathrm{CO}_{2}$. The cells at the logarithmic growth phase were harvested for the subsequent experiments when the cells reached $80 \%$ confluence.

RNA extraction and reverse transcription-quantitative polymerase chain reaction. Total RNA was extracted from samples and cell lines using TRIzol reagent (Invitrogen; Thermo Fisher Scientific, Inc.) according to the manufacturer's instructions. Subsequently, complementary DNA (cDNA) was synthesized from $2 \mu \mathrm{g}$ total RNA using the GoScript ${ }^{\mathrm{TM}}$ Reverse Transcription System kit (Promega Corporation, Madison, WI, USA) according to the manufacturer's instructions. Briefly, the samples were pre-incubated at $70^{\circ} \mathrm{C}$ for $10 \mathrm{~min}$, cooled on ice and then added to a reaction mixture consisting of $10 \mathrm{mmol} / \mathrm{l}$ deoxynucleotide triphosphate, $25 \mathrm{mmol} / 1 \mathrm{MgCl}_{2}$, 15 units avian myoblastosis virus reverse transcriptase, $10 \mathrm{X}$ reverse transcription buffer, 0.5 units $\mathrm{RNasin}^{\circledR}$ and $0.5 \mu \mathrm{g}$ oligo-(dT) 15 primer (all provided in the GoScript ${ }^{\mathrm{TM}}$ Reverse Transcription System kit). The reaction mixture (final volume, $20 \mathrm{ml}$ ) was incubated at $44^{\circ} \mathrm{C}$ for $15 \mathrm{~min}, 99^{\circ} \mathrm{C}$ for $5 \mathrm{~min}$ and $4^{\circ} \mathrm{C}$ for $5 \mathrm{~min}$. The cDNA was maintained at $20^{\circ} \mathrm{C}$ prior to use. The PCR reaction used $5 \mu \mathrm{g}$ cDNA. RT-qPCR was performed using SYBR Master mix [Takara Biotechnology (Dalian) Co. Ltd., Dalian, China] on a LightCycler 480 system (Roche Applied Science, Penzberg, Germany). The thermocycling conditions were as follows: 40 cycles of $94^{\circ} \mathrm{C}$ for $30 \mathrm{sec}$, $60^{\circ} \mathrm{C}$ for $30 \mathrm{sec}$ and $72^{\circ} \mathrm{C}$ for $30 \mathrm{sec}$. A human glyceraldehyde 3 -phosphate dehydrogenase (GAPDH) gene served as an endogenous control for sample normalization. Results were presented as the fold expression relative to that of GAPDH. PCR primers were as follows: Forward 5'-GAGTCAACG GATTTGGTCGT-3' and reverse 5'-GACAAGCTTCCCGTT CTCAG-3' for human GAPDH; and forward 5'-GGGAAT TCCCTGATTCCTCTTCA-3' and reverse 5'-CCCTCGAGC TAGAAGCTTACTTCCAT-3' for CIP2A. PCR products were separated by electrophoresis on $1.5 \%$ agarose gels. The gel images were captured with a camera and analyzed using NIH Image 2.0 (rsb.info.nih.gov/nih-image).

Western blot analysis. Total proteins from RPMI-8226 cells were extracted in lysis buffer (Beijing Solarbio Science \& Technology Co., Ltd., Beijing, China) and quantified using the Bradford method (Pierce Biotechnology, Inc., Rockford, IL, USA). Protein $(20 \mu \mathrm{g})$ was separated on $10 \%$ sodium dodecyl sulfate-polyacrylamide gel electrophoresis gels (Bio-Rad Labratories, Inc., Hercules, CA, USA), and transferred to nitrocellulose membrane (Bio-Rad Labratories, Inc.), and blocked by incubation with $5 \%$ non-fat milk in Tris-buffered saline with Tween-20 at room temperature for $1 \mathrm{~h}$. The membrane was then incubated overnight at $4^{\circ} \mathrm{C}$ with the following primary antibodies: Anti-CIP2A mouse monoclonal $\mathrm{IgG}_{1}$ (1:500; Santa Cruz Biotechnology, Inc., Santa Cruz, CA, USA; cat. no. sc-80662), rabbit monoclonal (PE Conjugate) c-Myc (D84C12) XP ${ }^{\circledR}[1: 1,000$; Cell Signaling Technology, Inc., Danvers, MA, USA (cat. no. 12189)], rabbit monoclonal Akt (1:1,000; Cell Signaling Technology, Inc.; cat. no. 4691), rabbit monoclonal p-Akt (Ser473) (D9E) XP ${ }^{\circledR}$ (1:2,000; Cell Signaling Technology, Inc.; cat. no. 4060) and rabbit polyclonal IgG anti-actin (H-196) (1:1,000; Santa Cruz Biotechnology, Inc.; cat. no. sc-7210). The membranes were washed with phosphate-buffered saline three times for $5 \mathrm{~min}$ each time. This was followed by incubation with horseradish peroxidase-conjugated goat anti-mouse (1:2,000; Santa Cruz Biotechnology, Inc.; cat. no. sc-2972) and goat anti-rabbit (1:2,000; Santa Cruz Biotechnology, Inc.; cat. no. sc-2030) secondary antibodies. Membranes were then washed again three times for 10 min each with TBST. Target protein bands were visualized using enhanced chemiluminescence (GE Healthcare Life Sciences, Chalfont, UK). All western immunoblot analyses were performed three times.

CIP2A short hairpin (sh)RNA transfection. The MM cells were seeded onto the 6 -well plates at a density of $2 \times 10^{5}$ cells per well prior to transfection. Sequences of CIP2A shRNA and shRNA control (Santa Cruz Biotechnology, Inc.) were as follows: 5'-AAACTTCTCTCAACATACTAGC-3' and 5'-CCTAAGGTTAAGTCGCCCTCG-3', respectively. Cells were plated in 6-well plates and infected with lentivirus constructs using $8 \mathrm{ng} / \mathrm{ml}$ polybrene (Santa Cruz Biotechnology, Inc.) as described in a previous study (10). The stable cell lines were selected in the presence of $1 \mu \mathrm{g} / \mathrm{ml}$ puromycin (Sigma-Aldrich, St. Louis, MO, USA) for 2 weeks.

Cell proliferation assay. An MTT assay was used to measure relative cell growth. Briefly, cells transduced shRNA for CIP2A and control shRNA were seeded into 96-well microplates $\left(3 \times 10^{3}\right.$ cells/well) for $48 \mathrm{~h}$ in RPMI-1640 medium with $10 \%$ FBS. After culture, cells were incubated with $20 \mu \mathrm{l} /$ well MTT (Sigma-Aldrich) at $37^{\circ} \mathrm{C}$ for $4 \mathrm{~h}$, and then $200 \mu 1$ dimethyl sulfoxide was added to each well. Cells were subjected to absorbance reading at $570 \mathrm{~nm}$ using a 96-well microplate reader (Synergy NEO; Bio-Tek Instruments, Inc., Winooski, VT, USA).

Colony formation assay. Cells were plated into three 6-cm cell culture dishes after transfection with CIP2A shRNA or control shRNA. Cells were incubated for two weeks in complete growth media. Cell colonies were fixed with cold methanol stained with $0.1 \%$ crystal violet (Beyotime Institute of Biotechnology, Haimen, China) for $30 \mathrm{~min}$. The colonies were manually counted using an inverted microscope (CKX41SF; Olympus Corporation, Tokyo, Japan).

Apoptosis analysis. At transfection, cells were harvested and washed with ice-cold PBS twice. Then, cells were re-suspended in Annexin V-binding buffer, and $5 \mu 1$ propidium iodide and $5 \mu \mathrm{l}$ Annexin V-fluorescein isothiocyanate (FITC; BD Pharmingen, San Diego, CA, USA) was added. Cells were analyzed by FACSCalibur flow cytometry (BD Biosciences, Franklin Lakes, NJ, USA). The proportion of apoptotic cells (Annexin V-positive cells) was presented as the mean \pm standard deviation.

Statistical analysis. All experiments were repeated at least three times. The data are presented as the mean \pm standard deviation and analyzed using SPSS software 15.0 (SPSS Inc., Chicago, IL, USA). Student's t-test was used to compare differences, and data were analyzed using the Pearson's $\chi^{2}$ test and Fisher's exact test. $\mathrm{P}<0.05$ and $\mathrm{P}<0.01$ were considered to indicate a statistically significant difference. 

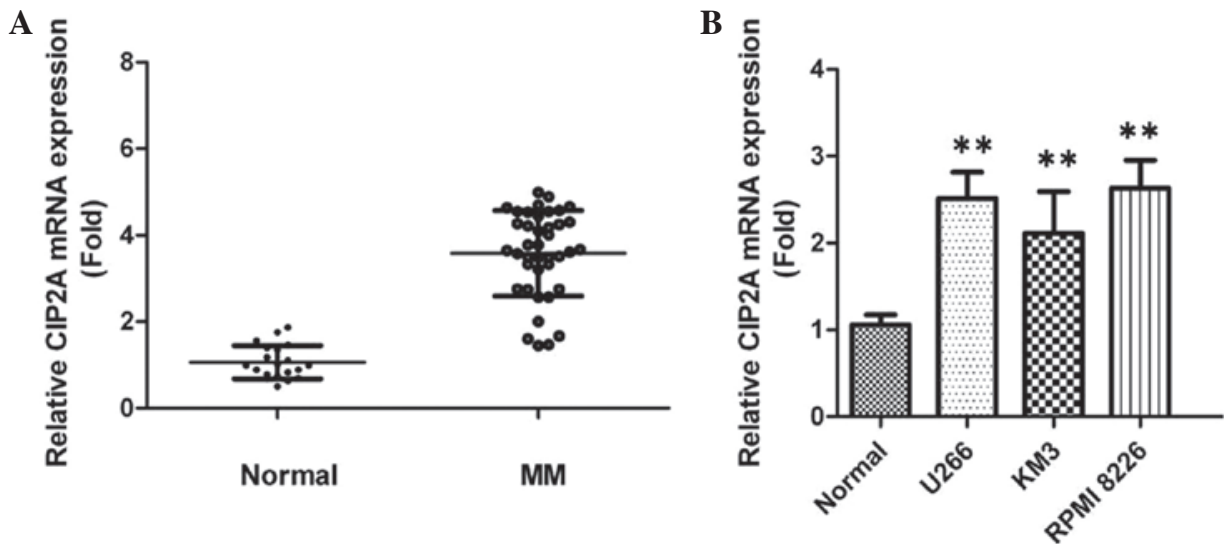

Figure 1. Reverse transcription-quantitative polymerase chain reaction showing expression level of CIP2A mRNA in (A) patients with MM and (B) in MM cell lines. ${ }^{* *} \mathrm{P}<0.01$. CIP2A, cancerous inhibitor of protein phosphatase $2 \mathrm{~A} ; \mathrm{MM}$, multiple myeloma.

\section{Results}

CIP2A is overexpressed in MM plasma and cell lines. The expression of CIP2A in the plasma of 40 patients with MM and 20 normal donors was examined by RT-qPCR. It was found that the level of CIP2A in plasma samples was upregulated in patients with MM compared with healthy control subjects (Fig. 1A), indicating that CIP2A may contribute to the development of MM. In addition, the expression level of CIP2A in MM cell lines was evaluated. Endogenous CIP2A was significantly increased with different levels in all of these MM cell lines, compared with normal plasma cells) from healthy donors (Fig. 1B). The RPMI-8226 cell line was used as the research model in the rest of the experiments.

CIP2A depletion in MM cell lines inhibits cell proliferation. The RPMI-8226 cell line was used to generate cell lines stably expressing CIP2A shRNA, in order to investigate the role of CIP2A in the pathogenesis of MM. The knockdown of CIP2A was confirmed by western blotting. CIP2A shRNA effectively decreased CIP2A expression in the RPMI-8226 cell line (Fig. 2A). The proliferation of RPMI-8226 cells was measured by an MTT assay. Targeting CIP2A with specific shRNA inhibited RPMI-8226 cell proliferation (Fig. 2B), suggesting the function of CIP2A in promoting MM cell proliferation. Furthermore, the role of CIP2A on proliferation was determined by colony formation assays. As shown in Fig. 3A and B, the total number of colonies and average colony diameter was markedly decreased with CIP2A knockdown. These findings suggested that CIP2A may regulate cell proliferation and potential tumorigenicity of MM.

CIP2A depletion induces apoptosis and inhibits c-Myc expression in MM cells. Annexin V-FITC analysis was used to detect the rate of apoptosis in RPMI-8226 cells. The results showed that CIP2A depletion could induce a notable population of early and late apoptotic RPMI-8226 cells compared with controls (Fig. 4). To investigate the mechanism underlying the effects of CIP2A, the c-Myc expression level was analyzed following knockdown of CIP2A. Western blot analysis revealed that CIP2A depletion downregulated the c-Myc protein expression in RPMI-8226 cells, indicating that CIP2A could regulate cell apoptosis and growth through the c-Myc signaling pathway (Fig. 2A).

\section{Discussion}

MM is an incurable disease characterized by plasma cell malignancy with heterogeneity (11). CIP2A is a tumor-associated antigen that has been identified in patients with different types of cancer. It has been reported that CIP2A is amplified and overexpressed in various solid and hematological tumors $(12,13)$, however, the role of CIP2A in patients with MM requires further investigation. Previous studies demonstrated cytoplasmic CIP2A overexpression in various types of cancer (14-16). In addition, Böckelman et al (17) noted that strong CIP2A expression could be associated with poor survival in ovarian cancer. The present study investigated the expression of CIP2A in plasma samples from patients with MM and normal donors, and found a significant upregulation of CIP2A in patients with MM, indicating that CIP2A may be important in MM. This result in patients was also in line with results in MM cell lines, which showed that endogenous CIP2A was significantly higher in all three MM cell lines compared with normal plasma cells from healthy donors. This suggests that CIP2A has a functional role in MM carcinogenesis.

CIP2A is a novel human oncoprotein that can protect c-Myc S62 from dephosphorylation and inhibit c-Myc associated protein phosphatase 2 activity (4). In the present study, our clinical investigation was extended and function studies in RPMI-8226 cells were conducted due to their high abundance of CIP2A. The results showed that the knockdown of CIP2A significantly decreased clonogenic formation and cell growth ability. The results demonstrated that CIP2A contributed to the colony formation and proliferation abilities of MM in vitro and may therefore represent a novel therapeutic target in patients with MM.

Apoptosis is regulated through two major pathways, the extrinsic and the intrinsic pathways, and these pathways can induce the activation of downstream effector caspases pathway. Recently, studies reported that the downregulation of CIP2A expression is associated with apoptosis in human cancer cells. In this study, the results showed that compared with control 

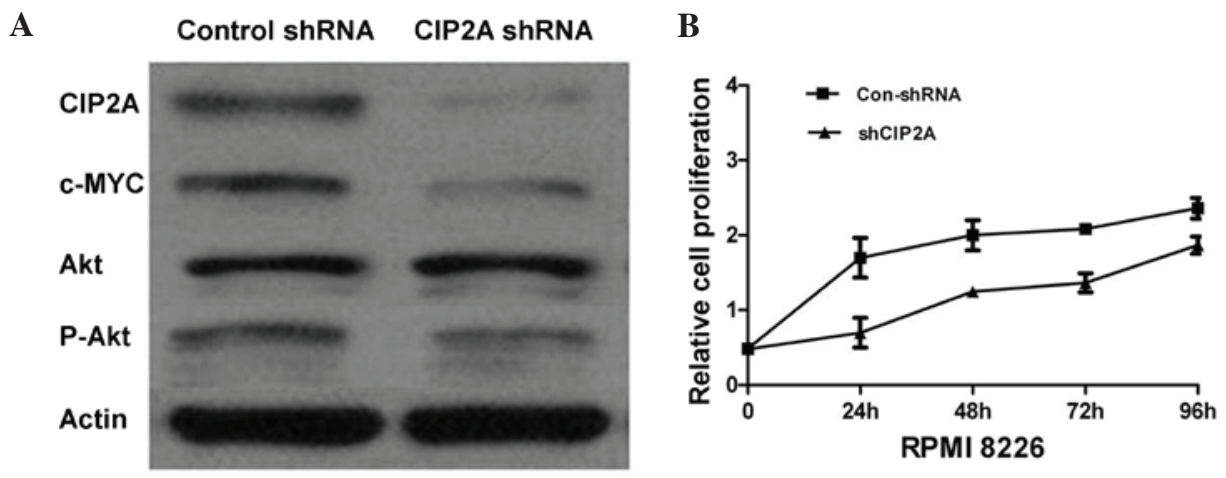

Figure 2. Effect of knocking down CIP2A on multiple myeloma cells. (A) Western blot analysis demonstrated that CIP2A shRNA treatment markedly decreased CIP2A levels and c-Myc levels in RPMI-8226 cells. (B) Knock down of CIP2A in RPMI-8226 cells decreased cell proliferation. CIP2A, cancerous inhibitor of protein phosphatase 2A; shRNA, short hairpin RNA; P-, phosphorylated.
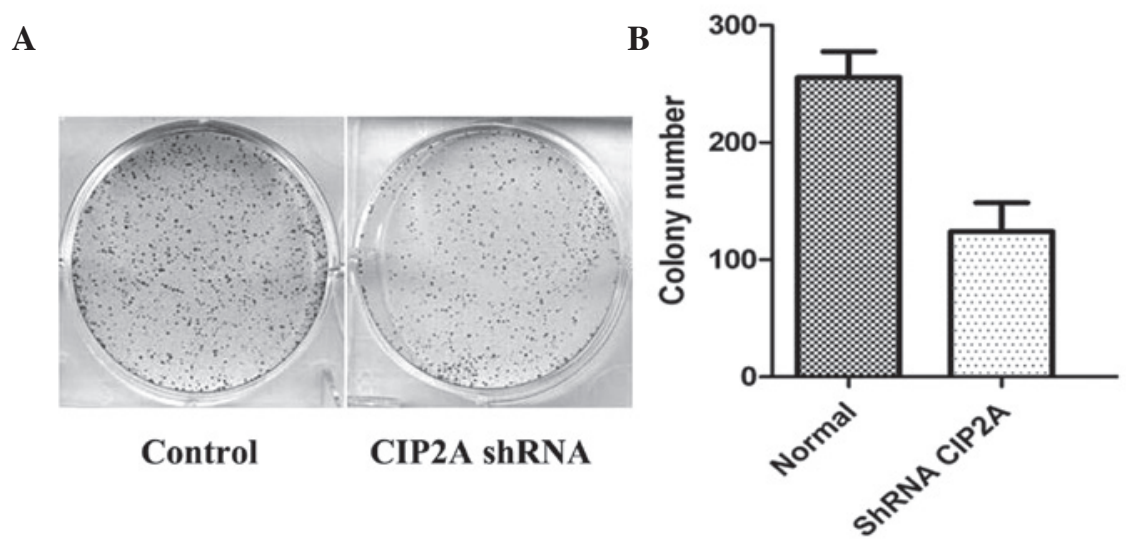

Figure 3. (A) Colony formation assay was performed in RPMI-8226 cells transfected with CIP2A shRNA. (B) A decrease was observed in the number of colonies in the groups treated with CIP2A shRNA. CIP2A, cancerous inhibitor of protein phosphatase 2A; shRNA, small hairpin RNA.

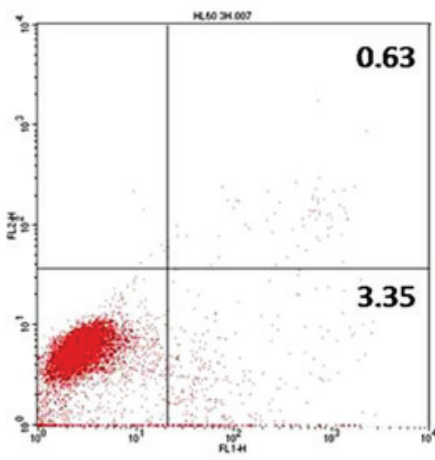

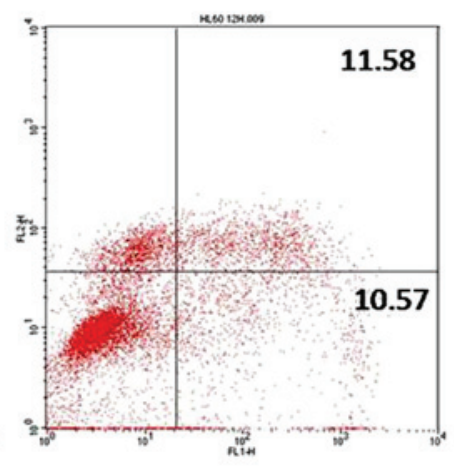

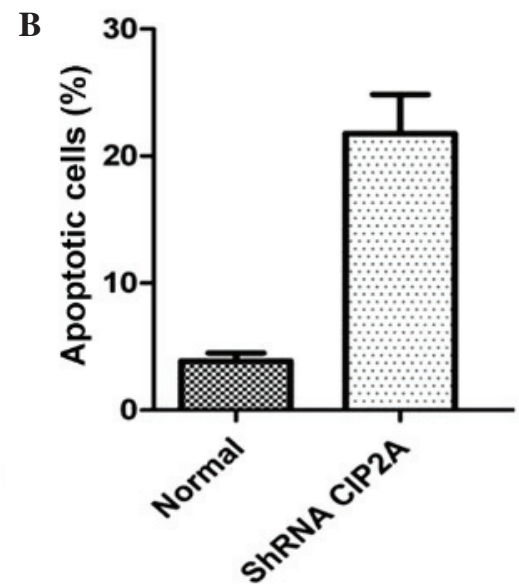

Figure 4. (A) Apoptotic cell death was determined by flow cytometric analysis with Annexin V and propidium iodide staining. RPMI-8226 cells were transfected with CIP2A shRNA and then subjected to an apoptosis assay. (B) The percentage of apoptosis, including early and late stage apoptotic cell death in each group, is shown. CIP2A, cancerous inhibitor of protein phosphatase 2A; shRNA, small hairpin RNA.

groups, CIP2A knockdown led to a significant increase in the apoptosis rate of RPMI-8226 cells, which suggests that inhibition of CIP2A may be a potential anticancer agent for patients with MM.

Previous studies revealed that CIP2A could regulate cancerous transformation and growth of cancer cells via
c-Myc signaling pathway regulation $(15,18)$. Furthermore, CIP2A promotes c-Myc expression, which is required for the proliferation of cancer cells. This study demonstrated that CIP2A silencing strongly suppresses cell proliferation by means of decreasing the expression of c-Myc and the level of AKT phosphorylation in vitro to a certain degree. 
In conclusion, CIP2A is upregulated in MM, and CIP2A expression promotes cell proliferation and clonogenic formation ability by regulating the expression of AKT phosphorylation and c-Myc. This study indicated that CIP2A may be important in the carcinogenesis and progression of MM.

\section{Acknowledgements}

This study was supported by the grants from the Science and Technology Research Projects of Shanxi Province (grant no. 20120313020-6).

\section{References}

1. Hatzimichael E, Dasoula A, Benetatos L, Syed N, Dranitsaris G, Crook T and Bourantas K: Study of specific genetic and epigenetic variables in multiple myeloma. Leuk Lymphoma 51: 2270-2274, 2010.

2. Richards T and Weber D: Advances in treatment for relapses and refractory multiple myeloma. Med Oncol 27 (Suppl 1): S25-S42, 2010.

3. Laubach JP, Richardson PG and Anderson KC: The evolution and impact of therapy in multiple myeloma. Med Oncol 27: S1-S6, 2010.

4. Khanna A, Böckelman C, Hemmes A, Junttila MR, Wiksten JP, Lundin M, Junnila S, Murphy DJ, Evan GI, Haglund C, et al: MYC-dependent regulation and prognostic role of CIP2A in gastric cancer. J Natl Cancer Inst 101: 793-805, 2009.

5. Johnson MD, Reeder JE, O'Connell M, Woodford M and Walter K: CIP2A and PP2A in human leptomeninges, arachnoid granulations and meningiomas. J Clin Neurosci 21: 2228-2232, 2014.

6. Li W, Ge Z, Liu C, Liu Z, Björkholm M, Jia J and Xu D: CIP2A is overexpressed in gastric cancer and its depletion leads to impaired clonogenicity, senescence, or differentiation of tumor cells. Clin Cancer Res 14: 3722-3728, 2008.

7. Junttila MR, Puustinen $P$, Niemelä $M$, Ahola R, Arnold H, Böttzauw T, Ala-aho R, Nielsen C, Ivaska J, Taya Y, et al: CIP2A inhibits PP2A in human malignancies. Cell 130: 51-62, 2007.
8. Choi YA, Park JS, Park MY, Oh KS, Lee MS, Lim JS, Kim KI, Kim KY, Kwon J, Yoon do Y, et al: Increase in CIP2A expression is associated with doxorubicin resistance. FEBS Lett 585: 755-760, 2011.

9. Terpos E, Morgan G, Dimopoulos MA, Drake MT, Lentzsch S, Raje N, Sezer O, García-Sanz R, Shimizu K, Turesson I, et al: International myeloma working group recommendations for the treatment of multiple myeloma-related bone disease. J Clin Oncol 31: 2347-2357, 2013.

10. Nasri M, Karimi A and Allahbakhshian Farsani M: Production, purification and titration of a lentivirus-based vector for gene delivery purposes. Cytotechnology 66: 1031-1038, 2014.

11. Zhan F, Huang Y, Colla S, Stewart JP, Hanamura I, Gupta S, Epstein J, Yaccoby S, Sawyer J, Burington B, et al: The molecular classification of multiple myeloma. Blood 108: 2020-2028, 2006.

12. Pang X, Fu X, Chen S, Zhu X, Qi H, Li Y, Li F and Tan W: Overexpression of CIP2A promotes bladder cancer progression by regulating EMT. Clin Transl Oncol 18: 289-295, 2016

13. Khanna A, Rane JK, Kivinummi KK, Urbanucci A, Helenius MA, Tolonen TT, Saramäki OR, Latonen L, Manni V, Pimanda $\mathrm{JE}$, et al: CIP2A is a candidate therapeutic target in clinically challenging prostate cancer cell populations. Oncotarget 6: 19661-19670, 2015.

14. Ventelä S, Sittig E, Mannermaa L, Mäkelä JA, Kulmala J, Löyttyniemi E, Strauss L, Cárpen O, Toppari J, Grénman R and Westermarck J: CIP2A is an Oct4 target gene involved in head and neck squamous cell cancer oncogenicity and radioresistance. Oncotarget 6: 144-158, 2015.

15. Junttila MR, Puustinen $P$, Niemelä $M$, Ahola R, Arnold $H$, Böttzauw T, Ala-aho R, Nielsen C, Ivaska J, Taya Y, et al: CIP2A inhibits PP2A in human malignancies. Cell 130: 51-62, 2007.

16. De P, Carlson J, Leyland-Jones B and Dey N: Oncogenic nexus of cancerous inhibitor of protein phosphatase 2A (CIP2A): An oncoprotein with many hands. Oncotarget 5: 4581-4602, 2014.

17. Böckelman C, Lassus H, Hemmes A, Leminen A, Westermarck J, Haglund C, Bützow R and Ristimäki A: Prognostic role of CIP2A expression in serous ovarian cancer. Br J Cancer 105: 989-995, 2011.

18. Yeh E, Cunningham M, Arnold H, Chasse D, Monteith T, Ivaldi G, Hahn WC, Stukenberg PT, Shenolikar S, Uchida T, et al: A signaling pathway controlling c-Myc degradation that impacts oncogenic transformation of human cells. Nat Cell Biol 6: 308-318, 2004. 